\title{
Et pourtant, Berne n'est pas si loin de la Romandie...
}

\section{Monique Gauthey}

Médecin spécialiste, ancien membre du comité central de la FMH

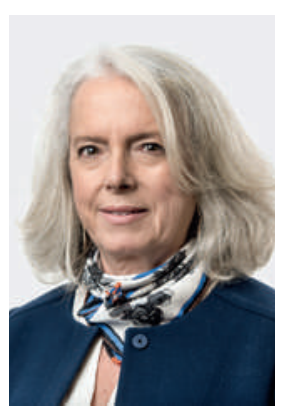

Après 8 ans de trajets parmi les nombreux pendulaires de la ligne Genève-Berne, le voyage m'a paru au fil du temps de plus en plus accessible, mais surtout j'ai mieux compris l'importance des actions quotidiennes conduites par la FMH.

Pourtant, peu de Genevois se sentent appelés vers la capitale fédérale. Le dialecte, qui n'est quasiment jamais brandi durant les séances de travail, exclut de fait beaucoup de Romands de toute complicité avec nos amis alémaniques. Cet handicap en décourage sûrement plus d'un... Vu depuis Genève, l'Europe semble bien plus proche et même les sursauts de la politique française résonnent à notre porte. J’ai observé à Berne les méandres de la politique fédérale, ses règles implicites et ses réseaux d'influence, et le poids des différentes régions.

\section{Vu depuis Genève, l'Europe semble bien plus proche que la capitale fédérale.}

En Suisse, un médecin sur 5 travaille dans un canton francophone et un sur 11 est en poste à Genève. L'orientation de Genève vers la France, ses liens avec le monde anglophone et avec les organisations internationales ajoutent encore aux spécificités de la médecine genevoise.

Mais les solutions politiques qui se construisent à Berne pour les médecins doivent s'appuyer sur les particularités de chaque région. La médecine de terrain est fortement marquée par le développement du tissu de santé régional, au fil de l'histoire de chaque canton. La FMH est une association plurielle, comme on en voit peu ailleurs en Europe, qui occupe tous les champs possibles, celui d'un ordre des médecins, d'un syndicat, d'un think tank politique et académique. Elle s'implique dans toutes sortes de domaines, éthique, déontologique, mais aussi pour la prévention, la qualité des prestations, les données statistiques ou de migration concernant les médecins. Ou encore d'autres domaines comme la facturation des actes médicaux, et même le développement du cadre légal qui touche à la santé. Il n'y a pas trop de toutes les forces vives qui la composent, entre le Comité central, l'Assemblée des délégués ou la Chambre médicale, pour faire avancer cet immense bateau.

La réduction du nombre de membres du Comité central de 9 à 7, effective depuis la dernière Chambre médicale du mois d'avril, a directement réduit la représentation des Romands et des Tessinois, mais elle reflète aussi un mouvement plus général, le passage du "citoyen se mobilisant pour l'intérêt général» à celui de «membre intéressé surtout à être consommateur de prestations», évolution délétère qu'on retrouve partout dans la société civile. La réduction à la fois du nombre de membres du Comité central, et du nombre de séances de l'Assemblée des délégués, a été décidée pour que les économies ainsi réalisées servent à des actions politiques urgentes. On passe ainsi d'une implication active de la part de nos membres à une politique passive, à l'écoute des effets possibles d'une campagne de presse.

Les solutions politiques doivent s'appuyer sur les particularités de la médecine de chaque région.

Ce virage subtil met les médecins romands en retrait, au moment où la complexification du domaine de la santé demanderait plus d'implication à tous les niveaux, dans toutes les commissions et tous les groupes de travail, nombreux autour de la FMH.

Ne pas avoir suffisamment de représentants romands dans nos organes de direction, c'est aussi se priver d'un réseau précieux de médecins, car chacun amène avec lui son carnet d'adresses.

$\mathrm{Au}$ moment de me retirer de la scène, je ne peux que plaider pour que les médecins romands se mobilisent, même s'il faut pour cela un peu forcer la porte de la FMH. 